Article

\title{
Jitter Evaluation in Distant and Adjacent Muscles after Botulinum Neurotoxin Type A Injection in 78 Cases
}

\author{
Joao Aris Kouyoumdjian ${ }^{1, * \mathbb{D}}$, Carla Renata Graça ${ }^{1}$ and Fabio Nazare Oliveira ${ }^{2}$ \\ 1 Laboratório Investigação Neuromuscular (LIN), Faculdade Estadual Medicina de São José do Rio \\ Preto (FAMERP), São José do Rio Preto SP 15090-000, Brazil; carlarenata@famerp.br \\ 2 Departamento de Ciências Neurológicas, Fundação Faculdade Regional de Medicina São José do Rio \\ Preto (FUNFARME), São José do Rio Preto SP 15090-000, Brazil; fabionazare@terra.com.br \\ * Correspondence: jaris@terra.com.br
}

Received: 16 July 2020; Accepted: 25 August 2020; Published: 27 August 2020

\begin{abstract}
To study the jitter parameters in the distant (DM) and the adjacent muscle (AM) after botulinum neurotoxin type A (BoNT/A) injection in 78 patients, jitter was measured by voluntary activation in DM $(n=43)$, and in AM $(n=35)$. Patients were receiving BoNT/A injections as a treatment for movement disorders. Mean age 65.1 years (DM) and 61.9 years (AM). The mean jitter was abnormal in $13.9 \%$ (maximum $41.4 \mu \mathrm{s}$ ) of DM, and $40 \%$ (maximum $43.7 \mu \mathrm{s}$ ) of AM. Impulse blocking was sparse. We found no correlation of the mean jitter to age, BoNT/A most recent injection (days/units), number of muscles injected, total BoNT/A units summated, number of total BoNT/A sessions, beta-blockers/calcium channel blockers use, and cases with local spread symptoms such as eyelid drop/difficulty swallowing. Maximum mean jitter (41.4/43.7 $\mu \mathrm{s})$ for DM/AM occurred 61 and 131 days since the most recent BoNT/A, respectively. The far abnormal mean jitter (32.6/36.9 $\mu \mathrm{s})$ occurred 229 and 313 days since the most recent BoNT/A. We suggested that jitter measurement can be done after BoNT/A in a given muscle other than the injected one, after 8 (DM) and 11 (AM) months, with reference $>33 \mu$ s and $>37 \mu$ s, respectively.
\end{abstract}

Keywords: single-fiber electromyography; jitter; botulinum neurotoxin type A; movement disorders; myasthenia gravis; neuromuscular junction

Key Contribution: (1) The mean jitter is increased in 13.9\% of distant muscle after botulinum neurotoxin type A injection, being a maximum of $41.4 \mu \mathrm{s}$. The mean jitter is increased by $40 \%$ of adjacent muscle after botulinum neurotoxin type A injection, being a maximum of $43.7 \mu \mathrm{s}$. (2) No correlation was found between mean jitter and the most recent botulinum neurotoxin type A injection (days/units).

\section{Introduction}

In 1977, Scott et at. [1], seeking a pharmacological alternative for strabismus correction, found an extraordinary benefit from a botulinum neurotoxin type A (BoNT/A) injection into the rectus muscles in adult rhesus monkeys. Since then, symptoms from many movement disorders have been greatly minimized from the BoNT/A injections. Botulinum neurotoxin high efficacy occurs because it has high specificity for skeletal and autonomic cholinergic nerves. It does not spread in high amounts outside the injection site, and it is poorly immunogenic [2,3].

Clostridium botulinum produces eight immunologically distinct serotypes (type A-H) that differ from each other by the nontoxic accessory protein (NAP) composition. For the clinical purpose, the BoNT/A is the most used and presented as onabotulinumtoxinA (ONA), abobotulinum toxin A 
(ABO), and incobotulinum toxin A (INCO) [4]. For pharmacological action, all should be cleaved from the NAP, except INCO that contains just the active 150-kD neurotoxin [4].

Botulinum neurotoxins enter the nerve terminals, where they cleave specifically to vesicle-associated membrane protein or synaptobrevin (VAMP), the synaptosomal associate protein of $25 \mathrm{kDa}$ (SNAP25) or syntaxin [5]. The proteins VAMP, SNAP25, and syntaxin in neuroexocytosis are collectively termed SNARE (SNAP REceptors) proteins because they were isolated as a receptor complex of the Soluble NSF Attachment Protein (SNAP) [5]. All BoNTs are expressed by two polypeptide chains (light $50 \mathrm{kDa}$, and heavy $100 \mathrm{kDa}$ ) linked by an interchain disulfide bond. If the light chain, which is the active part of the toxin, is present within a nerve terminal, the newly synthesized SNARE protein will continue to be cleaved [5]. The BoNT/B, BoNT/D, BoNT/F, and BoNT/G cleave VAMP. The BoNT/A and BoNT/E cleave SNAP25, and the BoNT/C cleaves both SNAP25 and syntaxin, resulting in the inhibition of acetylcholine release and paralysis [3].

Blocking neuromuscular transmission with BoNT/A, despite no injury to the motor axon, induces chemodenervation with a massive axon sprouting and neuromuscular junction remodeling [6,7]. Botulinum neurotoxin chemodenervation mimics axotomy, including the interruption of neurotransmission, induction of motor axon sprouting and upregulation of regeneration-associated proteins [8]. In rats, within a few days of the BoNT/A blocking, sprouts of the motor axons grow out over the surface of the muscle [7]. Around two weeks later, non-junctional or ectopic clusters of high acetylcholine receptor density begin to be settled. The effect of BoNT/A is long-lasting because the toxin irreversibly blocks the function of SNAP-25 molecules. However, a remarkable feature of the BoNT/A effect is its reversibility [5]. Recovery mechanisms include the formation of new proteins and axonal sprouting [9]. The neuromuscular block produced by the BoNT/A reaches its maximum in five days and, after that, remains stable for several months. After about ten days of BoNT, fibrillation potentials (FP) appeared and persisted throughout 60 days. The electromyographic findings in chronic botulinum poisoning are almost identical with those found following motor denervation, but in the last, the FP appears four to five days after the operation [10].

It is well known that the local effect of BoNT/A intramuscular injection either clinically (muscle paralysis) or through single-fiber electromyography (SFEMG) demonstrates very high jitter and impulse blocking [11-14]. The BoNT/A injection also may spread nearby, causing weakness, as seen in cervical dystonia (dysphagia) and in blepharospasm and hemifacial spasm (eyelid drop), that usually takes a few days to clear up [15]. Another defined point is the adjacent, and distant BoNT/A effect detected only through SFEMG with high jitter without or with sparse impulse blocking [11,16-26]. In a few cases, indisputable signs and symptoms of pseudo-botulism, such as weakness, blurred vision, diplopia, and difficulty swallowing, can occur after inaccurate dose injection. Finally, in very few cases, BoNT/A can precipitate or worsen symptoms from an unknown myasthenia gravis (MG) [27-29].

The reliability of the SFEMG jitter parameters in patients investigating neuromuscular junction disorders after receiving a BoNT/A injection is a challenge for the clinical neurophysiologists. From a practical perspective, there are no jitter reference values related to the time elapsed after the most recent BoNT/A injection obtained in a large patient cohort. The purpose here was to study the SFEMG jitter parameters in non-injected adjacent muscles and distant muscles from a large cohort of patients (78 cases) that have been in treatment with BoNT/A mostly for movement disorders. The objective is to find out the most reliable reference jitter parameters for those patients who had already been injected with BoNT/A and came to the SFEMG for a neuromuscular junction disorder suspicion.

\section{Results}

\subsection{Patients}

One patient with Alzheimer's disease was excluded from the study due to difficulties in getting spikes, remaining 78 cases. No patient complained of systemic symptoms like fatigue, blurred vision, or weakness, and they were very satisfied with the BoNT/A clinical results. Spread symptoms due to 
BoNT/A was far more common in cervical dystonia patients, where $57.1 \%$ complained of dysphagia ("difficulty swallowing") that lasted about 2 weeks; one case $(7.1 \%)$ had associated dysphonia. In cases of hemifacial spasm, eyelid drop occurred in $28.9 \%$ extending for a few days, and dry eye occurred in $6.7 \%$ of patients. In patients with blepharospasm, just one case $(7.7 \%)$ reported an eyelid drop. We found no relationship between cases with spread symptoms to the mean jitter values. Accordingly, the mean jitter was normal in the distant muscle (ED) in 5/7 (71.4\%), and in the adjacent muscle (Frontalis) in 11/17 (64.7\%) of those cases.

Most patients have comorbidities, and the mean daily pills used was 2.5, ranging from 0 to 11. Hypertension (35), depression (19), diabetes (10), hypothyroidism (9), and any arthritis (4) were the main associate disorders described by patients. History of atheromatous related-disorders, like chronic coronaropathy and mild stroke, were more difficult to obtain from patients, but some used antiplatelet medication. Any antihypertensive drug plus diuretics and beta-blockers represented the most used daily pills ( $n=70)$, followed by antidepressant drugs $(n=19)$, statins $(n=13)$, insulin, and metformin $(n=10)$, levothyroxine $(n=9)$, anticonvulsants $(n=7)$, and calcium channel blockers $(n=5)$. We checked specifically the relationship between the beta-blockers/calcium channel blockers to the mean jitter. From 22 patients that were on beta-blockers, 17 had normal and 5 an abnormal mean jitter. Of 5 patients in the use of calcium channel blockers, 4 had normal, and 1 had an abnormal mean jitter. So, we could not find any association that could act as a potential confusion factor.

The first group, to measure jitter parameters in a distant muscle, comprised 43 patients, age $65.1 \pm 10.5$ years (35 to 83 ), 70\% female. The patients were in the regular outpatient schedule for the BoNT/A injections due to hemifacial spasm (22), blepharospasm (10), cervical dystonia (6), aesthetics (2), bruxism (1), focal dystonia in the upper limb (1) and spasticity (1). BoNT/A was injected into one muscle (7 patients), two muscles (4 patients), three muscles (30 patients), and five muscles (2 patients). The most frequent injected muscles were Orbicularis Oculi (33), Risorius (21), Zygomaticus Major (21), Corrugator Supercilii (7), Sternocleidomastoideus (6), Splenius Capitis (6), Levator Scapulae (6), Orbicularis Oris (2), Procerus (2), Flexor Carpi Radialis (2), Trapezius (1), Masseter (1), Frontalis (1), Levator Nasalis (1), Platysma (1), Pectoralis Major (1), Biceps Brachii (1), Tibialis Posterior (1), and Extensor Pollicis Longus (1). In 2 patients, BoNT/A injections were made in the limb, but in these cases, the distant muscle tested was the contralateral one. In the remaining 41 patients, the muscles injected with BoNT/A were in the face or neck.

The second group, to measure jitter parameters in an adjacent muscle, comprised 35 patients, age $61.9 \pm 12.1$ years (30 to 79), 77\% female. The patients were in the regular outpatient schedule for the BoNT/A injections due to hemifacial spasm (23), cervical dystonia (6), blepharospasm (3), segmental dystonia (1), generalized dystonia (1), and cervical dystonia plus blepharospasm (1). Botulinum neurotoxin type A was injected into two muscles (5 patients), three muscles (25 patients), four muscles (4 patients), and five muscles (1 patient). The most frequent injected muscles were Orbicularis Oculi (28), Zygomaticus Major (24), Risorius (23), Sternocleidomastoideus (8), Splenius Capitis (8), Levator Scapulae (6), Corrugator Supercilii (4), Trapezius (3), and Masseter (1). In all cases, the muscles injected with BoNT/A were in the neck or the face.

The variables referring to the age, mean jitter, percentage of abnormal individual jitter values ("outliers"), the time elapsed (days) for SFEMG test since the first and most recent BoNT/A injection, the number of BoNT/A injections since the first one, the BoNT/A units injected per session, the total BoNT/A units summated since the first one, and the number of muscles injected per session are shown in Table 1 (distant group) and Table 2 (adjacent group). The table also shows the normality test for each one for the appropriate statistical tests. 
Table 1. Age, some jitter parameters, motor unit action potentials (MUAP) amplitude from the tested muscle, and some botulinum neurotoxin type A (BoNT/A) variables in the distant muscle group (43 patients).

\begin{tabular}{|c|c|c|c|c|c|c|c|c|}
\hline Variables & Normality-Test & Mean & SD & Min & Q1 & Median & Q3 & Max \\
\hline Age & Yes & 65.12 & 10.47 & 35 & & & & 83 \\
\hline $\begin{array}{l}\text { Mean jitter }(\mu \mathrm{s}) \\
\text { Abnormal }\end{array}$ & No & & & 17.1 & 20.7 & 23.5 & 26.2 & 41.4 \\
\hline $\begin{array}{l}\text { individual jitter } \\
\text { values }(\%)\end{array}$ & No & & & 0 & 0 & 5 & 5 & 30 \\
\hline Blocking (\%) & No & & & 0 & 0 & 0 & 0 & 5 \\
\hline BoNT/A first (days) & No & & & 0 & 1099 & 1743 & 2331 & 6575 \\
\hline BoNT/A last (days) & No & & & 5 & 64 & 85 & 138 & 229 \\
\hline $\begin{array}{l}\text { Number of } \\
\text { injections }\end{array}$ & $\mathrm{No}$ & & & 1 & 9 & 13 & 18 & 30 \\
\hline Units per injection & No & & & 30 & 60 & 60 & 80 & 600 \\
\hline Units total since first & No & & & 60 & 660 & 880 & 1440 & 9600 \\
\hline $\begin{array}{c}\text { Number muscles } \\
\text { injected }\end{array}$ & No & & & 1 & 2 & 3 & 3 & 5 \\
\hline
\end{tabular}

MIPI, mean inter-potential interval; SD, standard-deviation; Min, Minimum; Max, Maximum; Q1, Interquartile Range 1; Q3, Interquartile Range 3.

Table 2. Age, some jitter parameters, motor unit action potentials (MUAP) amplitude from the muscle, and some botulinum neurotoxin type A (BoNT/A) variables in the adjacent muscle group (35 patients).

\begin{tabular}{|c|c|c|c|c|c|c|c|c|}
\hline Variables & Normality-Test & Mean & SD & Min & Q1 & Median & Q3 & $\operatorname{Max}$ \\
\hline Age & Yes & 61.91 & 12.08 & 30 & & & & 79 \\
\hline Jitter $(\mu s)$ & No & & & 16.1 & 21.3 & 25.5 & 36.4 & 43.7 \\
\hline Abnormal & & & & & & & & \\
\hline $\begin{array}{l}\text { individual jitter } \\
\text { values }(\%)\end{array}$ & No & & & 0 & 5 & 10 & 25 & 70 \\
\hline Blocking (\%) & No & & & 0 & 0 & 0 & 5 & 15 \\
\hline BoNT/A first (days) & No & & & 238 & 1190 & 2050 & 3003 & 4365 \\
\hline BoNT/A last (days) & No & & & 11 & 42 & 82 & 182 & 320 \\
\hline $\begin{array}{l}\text { Number of } \\
\text { injections }\end{array}$ & Yes & 15.7 & 8.02 & 3 & & 9 & & 28 \\
\hline Units per injection & No & & & 45 & 60 & 60 & 200 & 550 \\
\hline Units total since first & No & & & 180 & 765 & 1200 & 1620 & \\
\hline $\begin{array}{c}\text { Number muscles } \\
\text { injected }\end{array}$ & No & & & 2 & 3 & 3 & 3 & 5 \\
\hline
\end{tabular}

MIPI, mean inter-potential interval; SD, standard-deviation; Min, Minimum; Max, Maximum; Q1, Interquartile Range 1; Q3, Interquartile Range 3.

The comparison between distant versus adjacent groups revealed no significant difference between age, the number of injections, the number of days from the first and the most recent injection of BoNT/A, BoNT/A units per injection, total BoNT/A units summed, and the number of muscles injected with BoNT/A per session (Table 3).

\subsection{Single-Fiber Electromyography}

In the distant group (43 patients), the muscles studied were Extensor Digitorum (40), Deltoideus (2), and Tibialis Anterior (1). In the adjacent group (35 patients), the only muscle studied was the Frontalis. The comparison between distant versus adjacent groups revealed a significant difference between the percentage of abnormal individual jitter values $(p=0.0003)$, and the percentage of impulse blocking $(p=0.0383)$ (Table 3). The mean jitter in the distant group was abnormal in 13.9\% (24.4 $\pm 5.28 \mu \mathrm{s})$, ranging from 17.1 to $41.1 \mu \mathrm{s}$, and impulse blocking was found in five cases $(11.6 \%)$. The mean jitter in the adjacent group was abnormal in $40 \%(28 \pm 8.2 \mu \mathrm{s})$, ranging from 16.1 to $43.7 \mu \mathrm{s}$, and impulse blocking was found 10 cases (28.6\%) (Figure 1 ). 
Table 3. Comparison between age, some jitter parameters, and some botulinum neurotoxin type A (BoNT/A) variables, from the distant and adjacent group.

\begin{tabular}{|c|c|c|c|c|}
\hline Variables & Distant vs. Adjacent & $t$-Test & $U$-Test & Significance \\
\hline Age & $p=0.2138$ & Yes & & No \\
\hline BoNT/A first (days) & $p=0.1689$ & & Yes & No \\
\hline BoNT/A last (days) & $p=0.9661$ & & Yes & No \\
\hline BoNT/A number of injections & $p=0.2785$ & Yes & & No \\
\hline BoNT/A Units per injection & $p=0.9633$ & & Yes & No \\
\hline BoNT/A Units total & $p=0.1153$ & & Yes & No \\
\hline Number of muscles injected & $p=0.0582$ & & Yes & No \\
\hline Mean jitter & $p=0.0723$ & & Yes & No \\
\hline $\begin{array}{c}\text { Abnormal individual jitter } \\
\text { values (\%) }\end{array}$ & $p=0.0003$ & & Yes & Yes \\
\hline Impulse blocking (\%) & $p=0.0383$ & & Yes & Yes \\
\hline
\end{tabular}

MIPI, mean inter-potential interval; $t$-test, Student's $t$-test; $U$-test, Mann-Whitney test.

Individual Value Plot of Mean Jitter: Distant and Adjacent

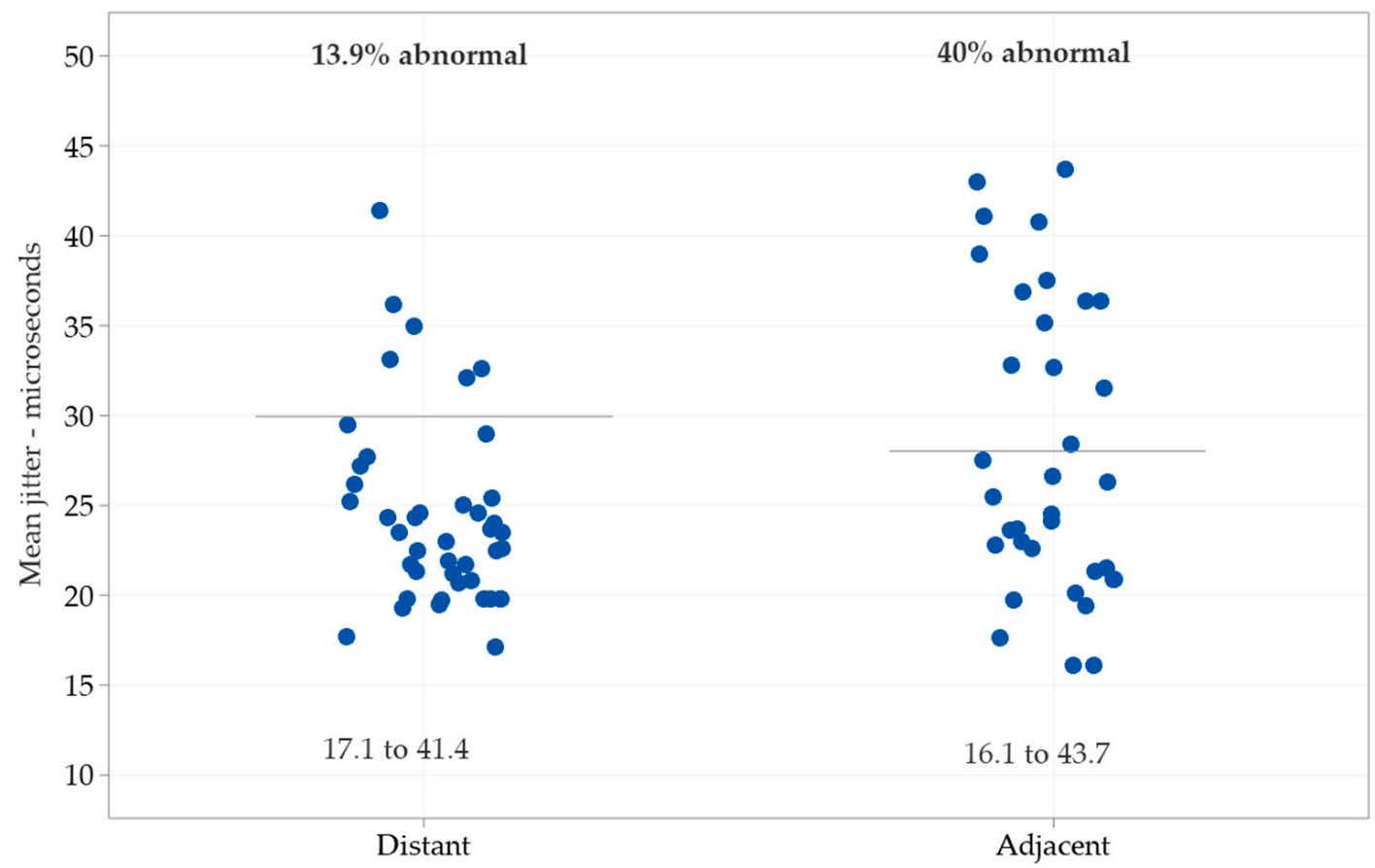

Figure 1. Individual value plot of the mean jitter in the distant $(n=43)$ compared to the adjacent group $(n=35)$. Observe the high proportion of increased jitter $(40 \%)$ in the adjacent versus only $13.9 \%$ in the distant group. Distant muscle, mostly Extensor Digitorum; adjacent muscle, all Frontalis.

\subsection{Correlation between Jitter and Variables}

The power of the correlation between the mean jitter values to some variables using a linear model and calculating the R-squared in percentiles were weak and ranged from $0 \%$ to $12.3 \%$ (Table 4 ). The correlation between age in the most recent BoNT/A injection versus mean jitter in the adjacent and distant muscle groups (A and B), and the number of days since the most recent BoNT/A injection versus mean jitter in the adjacent and distant muscle groups (C and D) are shown in Figure 2. 
Table 4. Correlation between the mean jitter values with some variables with a linear model (R-squared in percentiles) to show the correlation power in the regression line ( $0 \%$ no correlation; $100 \%$ strongest correlation).

\begin{tabular}{cccc}
\hline Variables & Distant Muscle & Adjacent Muscle & Power \\
\hline Mean jitter vs. BoNT/A units per injection & $12.8 \%$ & $5.9 \%$ & weak \\
Mean jitter vs. total BoNT/A units summated & $11.4 \%$ & $3.3 \%$ & weak \\
Mean jitter vs. days after last BoNT/A injection & $2.5 \%$ & $1.0 \%$ & weak \\
Mean jitter vs. age in the last BoNT/A injection & $2.3 \%$ & $5.3 \%$ & weak \\
Mean jitter vs. MUAP amplitude & $1.9 \%$ & $0.0 \%$ & weak \\
Mean jitter vs. number of injected muscles & $1.4 \%$ & $4.6 \%$ & weak \\
Mean jitter vs. days after first BoNT/A injection & $0.8 \%$ & $0.0 \%$ & very weak \\
\hline
\end{tabular}

BoNT/A, botulinum neurotoxin type A; MUAP, motor unit action potentials.
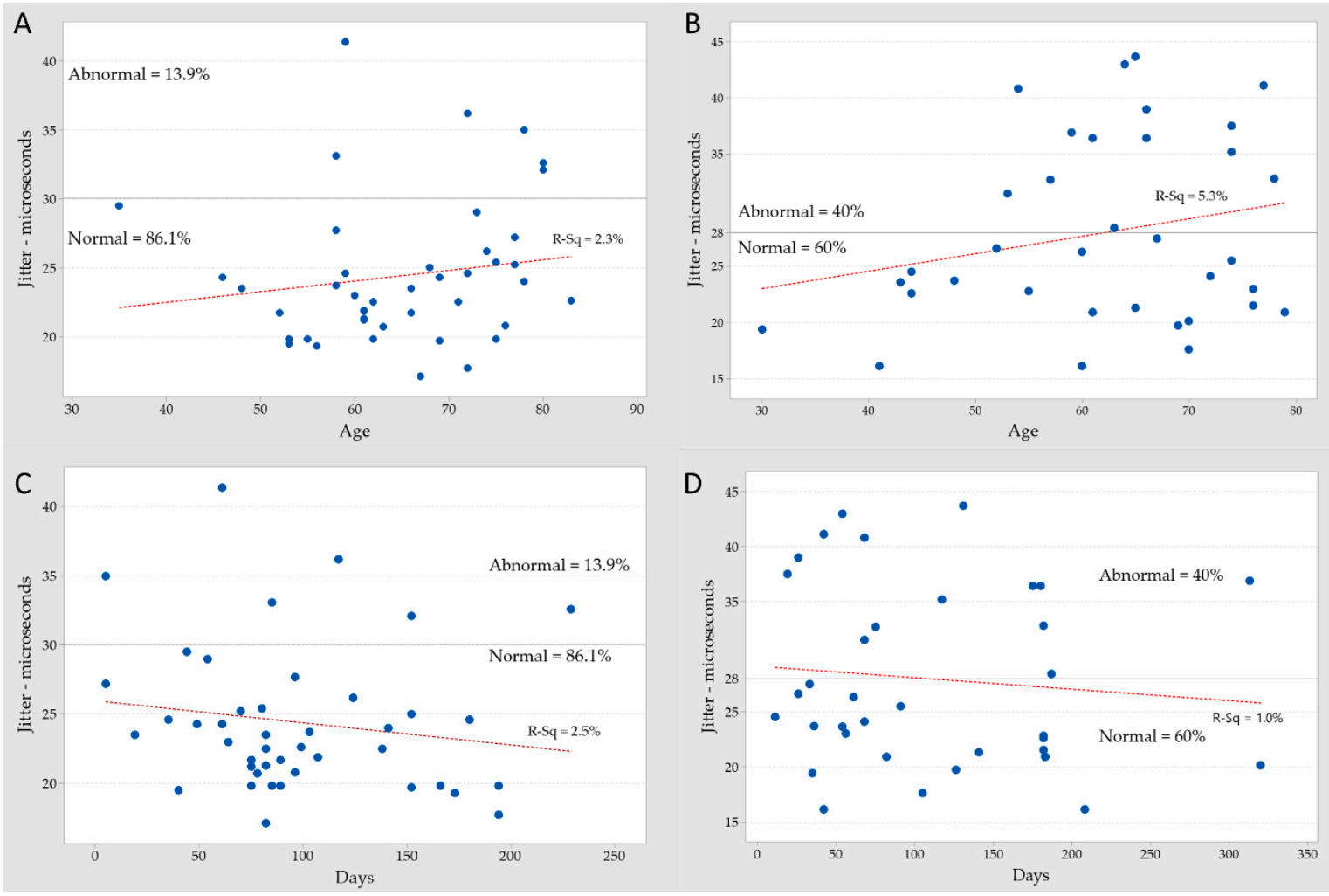

Figure 2. Correlation between the mean jitter and age in the most recent botulinum neurotoxin type A (BoNT/A) injection for the distant (A) and the adjacent muscle (B). Correlation between the mean jitter and days after the most recent BoNT/A injection in the distant (C) and the adjacent muscle (D).

\section{Discussion}

Our results revealed that a relatively mild mean jitter increased in $13.9 \%$ of patients from the distant group, in which the BoNT/A was injected mostly in facial or neck muscle, and the jitter was measured in a limb muscle, mostly ED. The maximum mean jitter obtained was $41.4 \mu \mathrm{s}(38 \%$ above the reference). For the patients in the adjacent group, in which the BoNT/A was injected mostly in facial or neck muscle and the jitter was measured in the Frontalis muscle, the mean jitter was increased by $40 \%$. The maximum mean jitter obtained was $43.7 \mu$ s ( $56.1 \%$ above the reference). We found a statistically significant difference in impulse blocking between groups, with this being more frequent in the adjacent group. However, there was no correlation statistically significant for any variable-age, days from the most recent BoNT/A injection, BoNT/A units in the most recent injection, total number of injections, and total BoNT/A summated since the first injection to predict the mean jitter increase. 
We specify the extreme findings for the mean jitter (lowest/highest) in Table 5, and the longest time that we had done the SFEMG test after the last BoNT/A injection in Table 6.

Table 5. The highest and lowest mean jitter related to age, sex, and the most recent botulinum neurotoxin type A (BoNT/A) injection (days and units), in the distant muscle (Extensor Digitorum), and in the adjacent muscle (Frontalis). M, male; F, female.

\begin{tabular}{ccccc}
\hline Variables & Distant & Distant & Adjacent & Adjacent \\
\hline Lowest mean jittert & & $17.1 \mu \mathrm{s}$ & & $16.1 \mu \mathrm{s}$ \\
Highest mean jitter & $41.4 \mu \mathrm{s}$ & & $43.7 \mu \mathrm{s}$ & \\
BoNT/A last & 61 days & 82 days & 131 days & 42 days \\
injection & $600 \mathrm{U}$ & $30 \mathrm{U}$ & $90 \mathrm{U}$ & $550 \mathrm{U}$ \\
Units & $51 \mathrm{M}$ & $67 \mathrm{~F}$ & $65 \mathrm{M}$ & $60 \mathrm{~F}$ \\
Age/Sex & &
\end{tabular}

Table 6. The longest time for jitter measurements after botulinum neurotoxin type A (BoNT/A) injection related to age, sex, and days/units since the most recent BoNT/A, in the distant muscle (Extensor Digitorum), and in the adjacent muscle (Frontalis).

\begin{tabular}{ccccc}
\hline Variables & Distant & Distant & Adjacent & Adjacent \\
\hline Lowest mean jitter & & $17.7 \mu \mathrm{s}$ & & $20.1 \mu \mathrm{s}$ \\
Highest mean jitter & $32.6 \mu \mathrm{s}$ & & $36.9 \mu \mathrm{s}$ & \\
BoNT/A last & 229 days & 194 days & 313 days & 320 days \\
injection & $60 \mathrm{U}$ & $60 \mathrm{U}$ & $400 \mathrm{U}$ & $45 \mathrm{U}$ \\
Units & $80 \mathrm{~F}$ & $72 \mathrm{M}$ & $59 \mathrm{M}$ & $70 \mathrm{M}$ \\
Age/Sex &
\end{tabular}

Even though both groups were composed of relatively older patients (mean age 65.1 and 61.9 for the distant and adjacent group, respectively), with more comorbidities associated, and consequently more daily medicine use, we could not find any evidence of age bias for the jitter parameters. There was no difference in the mean jitter from those taking beta-blockers or calcium channel blockers. None of the patients presented a systemic effect that could be attributed to pseudo-botulism, nor any symptoms from BoNT/A intolerance. All patients were very satisfied with the clinical results. We could not find any association between the cases who presented symptoms from the local BoNT/A spread (mainly dysphagia for cervical dystonia and eyelid drop for hemifacial spasm and blepharospasm) with the mean jitter value in both groups.

We found fifteen papers related to the local or distant effect of BoNT/A in humans [11-21,23,24,26,30]. The comparison was not an easy task due to the number of patients or healthy subjects, muscle injected, BoNT/A units used, time to do the SFEMG test, selection of patients (asymptomatic, fatigue or pseudo-botulism), and some variability in the adjacent or distant muscles studied for jitter parameters. Jitter parameters were studied in the same muscle injected in healthy subjects in a total of 102 muscles (Abductor Digiti Minimi, Extensor Digitorum Brevis, Extensor Digitorum, and Tibialis Anterior) [13,14,21], and also in the same muscle injected from patients (Orbicularis Oculi) in a total of 18 muscles [11,12]. The electrophysiological findings were reduced CMAP's amplitude (nadir at three weeks), no decrement at a slow frequency, no significant increment at $20 \mathrm{~Hz}$ (less than $10 \%$ ), FP more frequently found within the first six weeks [31], high jitter increase from 2 weeks until 8 weeks (89 to more than $300 \mu \mathrm{s}$ ), frequent impulse blocking, and no significative increase in fiber density [32]. The findings mentioned above were typical for chemodenervation and sometimes took many months to restore to normal.

We had selected reports in which the jitter parameters were studied in the adjacent or distant muscles from injected muscles or regions in healthy subjects or patients, provided that they do not have symptoms (fatigue or pseudo-botulism). The BoNT/A was injected into Orbicularis Oculi, glabellar region (Corrugator Supercillii and Procerus muscles), neck region, intragastric, detrusor, and 
various muscles in the face. The adjacent or distant muscle jitter studies were done mostly in Extensor Digitorum (87) and Orbicularis Oculi (32) [11,15-20,24,26,30]. It is likely that the ED muscles studied in the cases from Ruet et al. (2015) were the same as those described by Schnitzler et al. [24] As a whole, the mean jitter values were increased in distant muscle from none to $80 \%$. For the ED muscle, the mean jitter varied from 27 to $65 \mu$ s between 2 to 8 weeks since BoNT/A injection. For the Orbicularis Oculi muscle, 25.9 to $31.3 \mu$ s, between 2 to 8 weeks. For the Biceps Brachii muscle, 38 to $66 \mu \mathrm{s}$, between 4 to 6 weeks. No or rare impulse blocking was described. We consider the findings of Garner et al. [20] probably biased. They found remarkably increased jitter at week 4 (156 $\mu$ s) with $100 \%$ impulse blocking in the Extensor Digitorum Brevis muscle, which frequently has neurogenic findings.

The significant decline of CMAP amplitude, of both the Masseter and the Orbicularis Oris muscles after BoNT/A injection in the Orbicularis Oculi muscle suggests local instead of spreading axonal diffusion [33]. It also has been demonstrated that BoNT/A diffused through the fascia to the adjacent muscles [34]. Alimohammad et al. [15] emphasizes the physical spread of BoNT/A through small intramuscular vessels from the glabellar injection sites to the Orbicularis Oculi muscle. However, even in this well-controlled study, not all subjects had jitter values increased after two weeks since the injection. Garner et al. [20] did not find a correlation between the units of BoNT/A and the SFEMG findings. In disagreement, Girlanda et al. [19] concluded a BoNT/A dose-dependent effect on neuromuscular transmission in distant muscles. Sanders et al. [11] were more cautious about answering this issue and concluded that the local and distant effects of BoNT/A are "probably" dose-related. Our study did not find any correlation between the jitter parameters and the BoNT/A units (dose) used. We did not find a relationship with larger BoNT/A being expected to add distant jitter, as pointed out by Sanders et al. [11] The distant increased jitter is usually attributed to a systemic blood BoNT/A spread. In the present study, we could speculate an individual factor for BoNT/A reaching the muscle target on a distant limb since we find just $13.9 \%$ of patients with increased jitter. For the adjacent group, the "spread factor" is probably the operator's expertise to inject BoNT/A to the correct muscle motor point. As already mentioned, we had not found any isolated variable that could predict any direction to this question.

For the practical electrodiagnostic perspective for carrying out the SFEMG test for a MG suspicion, in a patient already injected with BoNT/A, we suggest reviewing the following rules. The major (and rare) systemic motor-autonomic symptoms related to the BoNT/A injection is the pseudo-botulism presented with diplopia, dysphagia, and dysarthria followed by a descending pattern of weakness affecting the upper limbs, then lower limbs, and in severe cases, respiratory muscle weakness, associated with blurred vision, constipation, dry mouth, postural hypotension, urinary retention, and pupillary abnormalities. The significant motor-autonomic symptoms related to the BoNT/A adjacent spread are dysphagia (it can extend for many weeks after neck muscles BoNT/A injection for cervical dystonia), eyelid drop (can take just a few days after Orbicularis Oculi BoNT/A injection), and dry eye. The unspecific fatigue symptom after BoNT/A should be seen with caution and clearly distinguished from the fluctuating weakness. If a given patient had a mean jitter greater than $37 \mu$ s for the Frontalis muscle (voluntary activation), and more than 11 months after the most recent BoNT/A injection, the increased jitter would probably be related to the MG. If a given patient had the mean jitter greater than $33 \mu$ for the Extensor Digitorum muscle (voluntary activation), more than eight months after the most recent BoNT/A injection, the increased jitter would probably be related to the MG. If a given patient had a mean jitter higher than $43 \mu \mathrm{s}$ (ED) and $45 \mu \mathrm{s}$ (Frontalis muscle) at any time, it will probably not be related to BoNT/A. The SFEMG test should never be done in the injected muscle, at any time.

There may be some possible minor limitations in this study. We did not measure the jitter parameters of the same patient at regular intervals after the BoNT/A injection due to the difficulty of having multiple SFEMG tests. However, this limitation was compensated by a large number of patients. Another minor limitation was the absence of reported CNE jitter reference for some muscles barely studied for the neuromuscular transmission suspicion disorders, as Deltoideus and Tibialis Anterior 
muscles. We consider it not a significant problem since the parameter variation for the reference jitter values obtained through CNE is much less than for SFE, and was used in 3 out of 43 muscles.

\section{Conclusions}

In conclusion, we found no cases with pseudo-botulism or fatigue symptoms. The percentage of cases with spread-related symptoms (dysphagia and eyelid drop) is the same as already reported. We found a jitter increase in a distant muscle from BoNT/A injection in 13.9\% of 43 patients, the highest value being $41.4 \mu \mathrm{s}$ (limit $30 \mu \mathrm{s}$ ). For the adjacent muscle from BoNT/A injection, we found a jitter increase in $40 \%$ of 35 patients, the highest value being $43.7 \mu$ s (limit $28 \mu$ s). There was sparse impulse blocking. There was no correlation between the mean jitter and several variables-days/units after the most recent BoNT/A injection, the number of muscles injected per session, the total number of BoNT/A sessions, total units summated since the first one, MUAP's amplitude, daily use of beta-blockers/calcium channel blockers, cases with symptoms from the local spread, and age. We presume that personal factors could be involved in the distant group and operator injection precision for the adjacent group. For electrodiagnostic practice, we suggested that jitter measurement can be analyzed after BoNT/A in a given muscle other than the injected, after eight (distant) and eleven (adjacent) months, with reference limit $>33 \mu$ s for and $>37 \mu$ s, respectively.

\section{Materials and Methods}

\subsection{Patients}

Between March 2018 to October 2019, 79 patients aged 30-83 years old, of both sexes, who routinely were injected with BoNT/A for movement disorders at the specific outpatient clinic of the Lucy Montoro Rehabilitation Center, Sao Jose do Rio Preto State Medical School (FAMERP), were recruited and invited to participate in the study. All cases were under the supervision of one of the authors (FO), the board-neurologist in charge of the diagnosis, and intramuscular injections of BoNT/A. Demographic data (sex and age), clinical data (diagnosis of movement disorder), comorbidities known to patients or described in the electronic medical records, drugs in continuous use, BoNT/A data (type, brand, the amount injected, periodicity, injected muscles, systemic adverse reactions, local adverse reaction, and result), time in days from the most recent BoNT/A injection to the SFEMG test, time in days from the first BoNT/A injection until the SFEMG test, and total dose (summated units) of BoNT/A until the SFEMG tests were done. The BoNT/A dose (units) was related to the muscle size. Abobotulinum toxin A (ABO; Dysport ${ }^{\circledR} /$ Ipsen Limited, Slough Berkshire, UK) was used in 77 patients. Only one patient has used three different BoNT/A injections over the years: Abobotulinum toxin A (ABO; Dysport ${ }^{\circledR} /$ Ipsen Limited, Slough Berkshire, UK), Incobotulinum toxin A (INCO; Xeomin ${ }^{\circledR} /$ Bocouture, Merz Pharmaceuticals GmbH, Frankfurt, Germany), and OnabotulinumtoxinA (ONA; Botox ${ }^{\circledR} /$ Vistabel, Allergan Inc., Irvine, CA, USA). The dilution was the same for all, $20 \mathrm{U}$ for every $0.1 \mathrm{~mL}$.

\subsection{Inclusion Criteria}

Patients with regular BoNT/A injections in the neck or facial muscles to measure the jitter in distant or adjacent muscle. Patients with regular BoNT/A injections in limb muscles to measure jitter in distant and contralateral muscle. Subjects with regular BoNT/A injections for aesthetic purposes in facial muscles provided that the jitter's measurement was done on a distant limb muscle.

\subsection{Exclusion Criteria}

Myasthenia gravis patients. Patients with previously confirmed neuromuscular diseases. Any weakness from known or unknown causes. Electromyographic evidence of active denervation or chronic reinnervation in the SFEMG tested muscle. Patients under 18 years of age. Patients without conditions to sustain minimal voluntary activation in the muscle studied. Patients in the use of anticoagulants. 


\subsection{Single-Fiber Electromyography}

The SFEMG test to measure jitter parameters was performed at the Neuromuscular Investigation Laboratory at the FAMERP by just one of the authors (JK), a neurologist, and a clinical neurophysiologist who was board-certified. All patients were regularly scheduled for BoNT/A injections for movement disorders, mostly for hemifacial spasm, blepharospasm, and cervical dystonia (together, 91\%); other disorders included bruxism, focal dystonia, generalized dystonia, spasticity, and aesthetics (together, 9\%). The Orbicularis Oculi, Zygomaticus Major, Risorius, Splenius Capitis, Sternocleidomastoideus, Levator Scapulae, and Corrugator Supercilii muscles represented 91\%. The Trapezius, Orbicularis Oris, Masseter, Procerus, Flexor Carpi Radialis, Platysma, Frontalis, Levator Nasalis, Pectoralis Major, Biceps Brachii, Tibialis Posterior, and Extensor Pollicis Longus muscles represented the remaining. Most of the patients had multiple muscles injected.

For the SFEMG test, patients were divided into two groups. In the first group (distant, 43 patients), the analysis was performed in a limb muscle, as follows, Extensor Digitorum $(n=40)$, Deltoideus $(n=2)$, and Tibialis Anterior $(n=1)$. In this group, the BoNT/A was injected in the neck or facial muscles (41 cases), in the forearm muscle (1 case being the SFEMG test performed in the opposite ED muscle) and in multiple muscles in one side of the body ( 1 case, being the SFEMG test performed in the opposing ED muscle). The choice of two other muscles instead of the ED was made when FPs were found, or the motor unit action potentials (MUAPs) revealed increased amplitude/duration (active denervation and chronic reinnervation, respectively) in the electromyography (EMG). In the second group (adjacent, 35 patients), the SFEMG test was performed in the Frontalis muscle in all cases. In these patients, the BoNT/A injection was performed in the neck or facial muscles, and not in the Frontalis muscle directly. The measurement of jitter parameters was done using the Natus ${ }^{\mathrm{TM}}$ Keypoint-Net (Middleton, WI, USA) or Natus ${ }^{\mathrm{TM}}$ UltraPro S100 (Middleton, WI, USA) machines, and an in-built software specifically developed for the SFEMG test. Before the SFEMG test, conventional non-quantitative EMG was performed in the chosen muscle to exclude neurogenic abnormalities.

The measurement of the jitter parameters by voluntary activation was performed using disposable concentric needle electrodes (CNE) of two brands: $\mathrm{Ambu}^{\circledR}$ Neuroline Concentric, $25 \mathrm{~mm} \times 0.30 \mathrm{~mm}$ (30 G), recording area $0.02 \mathrm{~mm}^{2}$ and, Natus ${ }^{\mathrm{TM}}$ Dantec ${ }^{\circledR}$ DCN Disposable Concentric Needle Electrode, $25 \mathrm{~mm} \times 0.30 \mathrm{~mm}(30 \mathrm{G})$, recording area $0.02 \mathrm{~mm}^{2}$. Both electrodes are routinely used and approved by the Brazilian Agency for Health Surveillance (ANVISA). The CNE was introduced into the chosen muscle. After minimal maintained voluntary contraction, the single fiber action potentials (SFAPs), or more appropriately, the "apparent single fiber action potentials" (ASFAPs) were recorded. Due to the use of disposable CNE, with a much larger recording area than the single fiber electrode, the probability of recording spikes from two or more muscle fibers increased; thus, the term ASFAP is better for defining these potentials. The low-frequency filter was set at $1 \mathrm{kHz}$ to suppress the distant action potentials (APs) from the CNE recording area, passing only the APs with rapid rise time. Because the low-frequency filter reduces the amplitude of APs, we accepted only the ones with a minimum amplitude limit of $100 \mu \mathrm{V}$. Records were made after a minimum of three CNE insertions with radial advancement into the muscle. After slight movements of the electrode, a pair of ASFAPs was recorded, with both potentials belonging to the same motor unit; the trigger was put to the higher amplitude spike to get the temporal variation on the second smaller spike.

The jitter value was calculated as the mean consecutive differences (MCD) for each pair, ideally for 100 pairs; in some circumstances, we accepted a minimum of 30 pairs. The MCD variation was calculated by the "amplitude level", i.e., by the temporal variation of the ascending lines of depolarization of the ASFAPs. Acceptable potentials were based on the criteria established in the literature [11], as follows: parallel depolarization ascending lines; similar shape in the superimposition of potentials; absence of summation characterized by notches and shoulders in the APs depolarization lines; a regular peak of the APs. Small amplitude variations were tolerated. We excluded ASFAP pairs without clear separation between them, records with less than 30 ASFAP pairs, ASFAP pairs with interpotential intervals (IPI) greater than $4 \mathrm{~ms}$ to avoid the effect of velocity recovery function (VRF), 
or when the shape of potentials did not remain constant in consecutive discharges. The classic SFAPs presents rise-time $<300 \mu$ s and the same shape/amplitude in consecutive discharges, quickly confirmed in the superimposition of at least ten potentials and, APs pairs must have a clear baseline separation.

The test conclusion was defined by the mean of 20 different MCDs for the reference value of $30 \mu \mathrm{s}$ for the ED, Deltoideus and Tibialis Anterior muscles, and $28 \mu$ s for the Frontalis muscle [35]. Although the multicenter study for the new reference values for $\mathrm{CNE}$ jitter parameters did not contemplate large muscles other than the Extensor Digitorum, we use its reference for the Deltoideus muscle (use here in 2 cases) and the Tibialis Anterior muscle (used here in 1 case). The mean value of the sorted differences (MSD) was also obtained. MSD calculates the consecutive differences according to the frequency of discharge of the potential pairs. When the inter-discharge interval (IDI) variation is larger than $4 \mathrm{~ms}$ and not constant, there may be interference from the VRF effect. The mean jitter was calculated by the mean MCD or MSD, which had the lowest value for each pair. Mean jitter values higher than $150 \mu \mathrm{s}$ were fixed at this value, to minimize the bias due to the VRF effect; the higher the jitter, the greater the probability of impulse blocking, consequently further artificially adding jitter. The other way for the test conclusion was calculated as the percentage of the individual MCDs above the reference limit $(43 \mu \mathrm{s})$; when more than $10 \%$ of the ASFAP pairs, i.e., at least 3 out of 20 , have values higher than $43 \mu \mathrm{s}$ for the ED, Deltoideus and Tibialis Anterior muscles and, $38 \mu$ for the Frontalis muscle, the test was abnormal. Impulse blocking was considered present or absent, regardless of the percentage in each pair. The skin temperature was maintained at more than $30^{\circ} \mathrm{C}$.

\subsection{Statistics}

Descriptive statistics calculated the mean jitter (MCD) values of the 78 cases and all other parameters: mean and standard deviation for normal or Gaussian distribution or median/percentage for non-normal distribution. An Anderson-Darling normality test was used. The Student's $t$-test made the comparison of parametric variables with a significance of 0.05 . The comparison of nonparametric variables was made by the Mann-Whitney nonparametric test ( $U$-test) to verify the medians' equality. Correlation of the mean jitter values (MCD) with variables was made with a linear model, calculating the R-squared in percentiles ranging from 0 to $100 \%$ to show the correlation power of jitter in the regression line.

\subsection{Ethics}

The study was in accord with the Helsinki Declaration of 1975 and approved by the ethics committee of the Faculdade de Medicina de São José do Rio Preto, São Paulo, Brazil, where the SFEMG tests were performed. Certificate of Presentation for Ethical Consideration (CAAE) approved number 83336818.0.0000.5415 on 22 March 2018. All patients signed informed consent.

Author Contributions: Conceptualization, J.A.K., C.R.G. and F.N.O.; methodology, J.A.K., C.R.G. and F.N.O.; formal analysis, J.A.K. and C.R.G.; investigation, J.A.K.; writing-original draft preparation, J.A.K. and C.R.G.; writing-review and editing, J.A.K. and C.R.G.; supervision, J.A.K. All authors have read and agreed to the published version of the manuscript.

Funding: This research received no external funding.

Conflicts of Interest: The authors declare no conflict of interest.

\section{References}

1. Scott, A.B.; Rosenbaun, A.; Collins, C.C. Pharmacologic weakening of extraocular muscles. Investig.Ophthalmol. Vis. Sci. 1973, 12, 924-927.

2. Rossetto, O.; Megighian, A.; Scorzeto, M.; Montecucco, C. Botulinum neurotoxins. Toxicon 2013, 67, 31-36. [CrossRef] [PubMed]

3. Rossetto, O.; Pirazzini, M.; Montecucco, C. Botulinum neurotoxins: Genetic, structural, and mechanistic insights. Nat. Rev. Microbiol. 2014, 12, 535-549. [CrossRef] [PubMed] 
4. Scaglione, F. Conversion ratio between Botox ${ }^{\circledR}$, Dysport ${ }^{\circledR}$, and Xeomin ${ }^{\circledR}$ in clinical practice. Toxins (Basel) 2016, 8, 65. [CrossRef]

5. Pantano, S.; Montecucco, C. The blockade of the neurotransmitter release apparatus by botulinum neurotoxins. Cell. Mol. Life Sci. 2014, 71, 793-811. [CrossRef]

6. Hong, B.; Chen, M.; Hu, X. Influence of injection of chinese botulinum toxin type A on the histomorphology and myosin heavy chain composition of rat gastrocnemius muscles. J. Zhejiang Univ. Sci. B 2013, 14, 983-992. [CrossRef]

7. Slater, C.R. 'Fragmentation' of NMJs: A sign of degeneration or regeneration? A long journey with many junctions. Neuroscience 2020, 439, 28-40. [CrossRef]

8. Franz, C.K.; Puritz, A.; Jordan, L.A.; Chow, J.; Ortega, J.A.; Kiskinis, E.; Heckman, C.J. Botulinum toxin conditioning enhances motor axon regeneration in mouse and human preclinical models. Neurorehabil. Neural Repair 2018, 32, 735-745. [CrossRef]

9. Schulte-Mattler, W.J. Use of botulinum toxin A in adult neurological disorders: Efficacy, tolerability, and safety. CNS Drugs 2008, 22, 725-738. [CrossRef]

10. Josefsson, J.-O.; Thesleff, S. Electromyographic findings in experimental botulinum intoxication. Acta Physiol. Scand. 1961, 51, 163-168. [CrossRef]

11. Sanders, D.B.; Massey, E.W.; Buckley, E.G. Botulinum toxin for blepharospasm: Single-fiber EMG studies. Neurology 1986, 36, 545-547. [CrossRef] [PubMed]

12. Bogucki, A. Serial SFEMG studies of orbicularis oculi muscle after the first administration of botulinum toxin. Eur. J. Neurol. 1999, 6, 461-467. [CrossRef] [PubMed]

13. Lispi, L.; Leonardi, L.; Petrucci, A. Longitudinal neurophysiological assessment of intramuscular type-A botulin toxin in healthy humans. Neurol. Sci. 2018, 39, 329-332. [CrossRef] [PubMed]

14. Eleopra, R.; Rinaldo, S.; Montecucco, C.; Rossetto, O.; Devigili, G. Clinical duration of action of different botulinum toxin types in humans. Toxicon 2020, 179, 84-91. [CrossRef]

15. Alimohammadi, M.; Andersson, M.; Punga, A.R. Correlation of botulinum toxin dose with neurophysiological parameters of efficacy and safety in the glabellar muscles: A double-blind, placebo-controlled, randomized study. Acta Derm. Venereol. 2014, 94, 32-37. [CrossRef]

16. Lange, D.J.; Brin, M.F.; Warner, C.L.; Fahn, S.; Lovelace, R.E. Distant effects of local injection of botulinum toxin. Muscle Nerve 1987, 10, 552-555. [CrossRef]

17. Olney, R.K.; Aminoff, M.J.; Gelb, D.J.; Lowenstein, D.H. Neuromuscular effects distant from the site of botulinum neurotoxin injection. Neurology 1988, 38, 1780-1783.

18. Lange, D.J.; Rubin, M.; Greene, P.E.; Kang, U.J.; Moskowitz, C.B.; Brin, M.F.; Lovelace, R.E.; Fahn, S. Distant effects of locally injected botulinum toxin: A double-blind study of single fiber EMG changes. Muscle Nerve 1991, 14, 672-675. [CrossRef]

19. Girlanda, P.; Vita, G.; Nicolosi, C.; Milone, S.; Messina, C. Botulinum toxin therapy: Distant effects on neuromuscular transmission and autonomic nervous system. J. Neurol. Neurosurg. Psychiatry 1992, 55, 844-845. [CrossRef]

20. Garner, C.G.; Straube, A.; Witt, T.N.; Gasser, T.; Oertel, W.H. Time course of distant effects of local injections of botulinum toxin. Mov. Disord. 1993, 8, 33-37. [CrossRef]

21. Wan, X.; Tang, X.; Cui, L. Remote effects of local injection of botulinum toxin type A. Zhongguo Yi Xue Ke Xue Yuan Xue Bao 1999, 21, 362-367. [PubMed]

22. Comella, C.L.; Pullman, S.L. Botulinum toxins in neurological disease. Muscle Nerve 2004, $29,628-644$. [CrossRef] [PubMed]

23. Roche, N.; Schnitzler, A.; Genêt, F.; Durand, M.-C.; Bensmail, D. Undesirable distant effects following botulinum toxin type a injection. Clin. Neuropharmacol. 2008, 31, 272-280. [CrossRef] [PubMed]

24. Schnitzler, A.; Genet, F.; Durand, M.-C.; Roche, N.; Bensmail, D.; Chartier-Kastler, E.; Denys, P. Pilot study evaluating the safety of intradetrusor injections of botulinum toxin type A: Investigation of generalized spread using single-fiber EMG. Neurourol. Urodyn. 2011, 30, 1533-1537. [CrossRef] [PubMed]

25. Thomas, A.M.; Simpson, D.M. Contralateral weakness following botulinum toxin for poststroke spasticity. Muscle Nerve 2012, 46, 443-448. [CrossRef] [PubMed]

26. Ruet, A.; Durand, M.-C.; Denys, P.; Lofaso, F.; Genet, F.; Schnitzler, A. Single-fiber electromyography analysis of botulinum toxin diffusion in patients with fatigue and pseudobotulism. Arch. Phys. Med. Rehabil. 2015, 96, 1103-1109. [CrossRef] 
27. Watts, J.; Brew, B.; Tisch, S. Myasthenia gravis exacerbation with low dose ocular botulinum toxin for epiphoria. J. Clin. Neurosci. 2015, 22, 1979-1981. [CrossRef]

28. Timmermans, G.; Depierreux, F.; Wang, F.; Hansen, I.; Maquet, P. Cosmetic injection of botulinum toxin unmasking subclinical myasthenia gravis: A case report and literature review. Case Rep. Neurol. 2019, 11, 244-251. [CrossRef]

29. Punga, A.R.; Liik, M. Botulinum toxin injections associated with suspected myasthenia gravis: An underappreciated cause of MG-like clinical presentation. Clin. Neurophysiol. Pract. 2020, 5, 46-49. [CrossRef]

30. Osio, M.; Mailland, E.; Muscia, F.; Nascimbene, C.; Vanotti, A.; Bana, C.; Corsi, F.; Foschi, D.; Mariani, C. Botulinum neurotoxin-A does not spread to distant muscles after intragastric injection: A double-blind single-fiber electromyography study. Muscle Nerve 2010, 42, 165-169. [CrossRef]

31. Ostergaard, L.; Fuglsang-Frederiksen, A.; Werdelin, L.; Sjö, O.; Winkel, H. Quantitative EMG in botulinum toxin treatment of cervical dystonia. A double-blind, placebo-controlled study. Electroencephalogr. Clin. Neurophysiol. 1994, 93, 434-439. [CrossRef]

32. Odergren, T.; Tollbäck, A.; Borg, J. Electromyographic single motor unit potentials after repeated botulinum toxin treatments in cervical dystonia. Electroencephalogr. Clin. Neurophysiol. 1994, 93, 325-329. [CrossRef]

33. Eleopra, R.; Tugnoli, V.; Caniatti, L.; De Grandis, D. Botulinum toxin treatment in the facial muscles of humans: Evidence of an action in untreated near muscles by peripheral local diffusion. Neurology 1996, 46, 1158-1160. [CrossRef] [PubMed]

34. Shaari, C.M.; George, E.; Wu, B.L.; Biller, H.F.; Sanders, I. Quantifying the spread of botulinum toxin through muscle fascia. Laryngoscope 1991, 101, 960-964. [CrossRef] [PubMed]

35. Stålberg, E.; Sanders, D.B.; Ali, S.; Cooray, G.; Leonardis, L.; Löseth, S.; Machado, F.; Maldonado, A.; Martinez-Aparicio, C.; Sandberg, A.; et al. Reference values for jitter recorded by concentric needle electrodes in healthy controls: A multicenter study. Muscle Nerve 2016, 53, 351-362. [CrossRef] [PubMed]

(C) 2020 by the authors. Licensee MDPI, Basel, Switzerland. This article is an open access article distributed under the terms and conditions of the Creative Commons Attribution (CC BY) license (http://creativecommons.org/licenses/by/4.0/). 